\title{
La enseñanza remota en la autonomía para el aprendizaje de estudiantes de universidades latinoamericanas
}

\section{Remote teaching in the learning autonomy of Latin American university students}

Miguel Sebastián Armesto-Céspedes

Universidad Peruana de Ciencias Aplicadas, Lima, Perú

miguel.armesto@upc.edu.pe

https://orcid.org/0000-0003-0497-0891

Rony Isaac Vallejos-Armas

Universidad Peruana de Ciencias Aplicadas, Lima, Perú

rvallejo@upc.edu.pe

https://orcid.org/0000-0001-8258-1109

Daniela Medina-Coronado

Universidad César Vallejo, Lima, Perú

dmedinac@ucv.edu.pe

https://orcid.org/0000-0002-9180-7613

Karina Raquel Bartra-Rivero

Universidad Peruana de Ciencias Aplicadas, Lima, Perú

pchukbar@upc.edu.pe

(D) https://orcid.org/ 0000-0002-9686-2347

Recepción: 02/08/2021 | Aceptación: 09/11/2021 | Publicación: 25/11/2021

Cómo citar (APA, séptima edición):

Armesto-Céspedes, M.S., Vallejos-Armas, R. I., Medina-Coronado, D., y Bartra-Rivero, K. R. (2021). La enseñanza remota en la autonomía para el aprendizaje de estudiantes de universidades latinoamericanas. INNOVA Research Journal, 6(3.2), 1-13. https://doi.org/10.33890/innova.v6.n3.2.2021.1876

\section{Resumen}

La educación entendida como un proceso en el que la sociedad proyecta sus valores, roles y traslada conocimiento para que sus ciudadanos puedan aplicarlas de la mejor manera para mejorar su entorno y calidad de vida, cuenta con sus propios modelos, estrategias y medios para poder 
llevar a cabo el proceso formativo. Sin embargo, estas se han adecuado ante los cambios y circunstancias adversas producto de la pandemia producida por el COVID-19. Entre estos cambios, la virtualidad evidenciada en la educación remota se ha erigido como una solución real dentro de este contexto. El mundo ha tenido que adaptarse, y Latinoamérica se ha enfrentado a la pandemia con sus modelos, estructuras y actores educativos como mejor ha podido, obteniendo resultados e impactos diversos. El objetivo del presente artículo está orientado a analizar las experiencias desarrolladas por las universidades de diversos países de la región y su impacto en la autonomía en el aprendizaje en estudiantes del pregrado, en el marco de la enseñanza remota por emergencia. Para su desarrollo, se ha recurrido a la revisión de diversos artículos de bases de datos de acceso abierto en español y publicadas durante el periodo de pandemia. Entre los resultados más relevantes se aprecia que la planificación para la enseñanza y la acción docente son claves para que los estudiantes aprendan de forma significativa y se promuevan las habilidades para la autogestión de los aprendizajes, en aquellas experiencias de réplica de la enseñanza presencial, no se observan resultados auspiciosos.

Palabras claves: pandemia; educación remota; autonomía en el aprendizaje.

\section{Abstract}

Education, understood as a process in which society projects its values, roles and transfers knowledge so that its citizens can apply them in the best way to improve their environment and quality of life, has its own models, strategies and means to carry out the educational process. However, these have been adapted to the changes and adverse circumstances resulting from the COVID-19 pandemic. Among these changes, the virtuality of remote education has emerged as a real solution in this context. The world has had to adapt, and Latin America has faced the pandemic with its educational models, structures and actors as best it can, obtaining diverse results and impacts. The aim of this article is to analyse the experiences developed by universities in various countries in the region and their impact on the learning autonomy of undergraduate students, within the framework of emergency remote teaching. For its development, we have resorted to the review of various articles in open access databases in Spanish and published during the pandemic period. Among the most relevant results, it can be seen that planning for teaching and teaching actions are key for students to learn in a meaningful way and to promote skills for self-management of learning, in those experiences of replication of face-to-face teaching, auspicious results are not observed.

Keywords: pandemic; remote education; learning autonomy.

\section{Introducción}

En lo que se lleva de este siglo, la humanidad ha sido testigo de una de las crisis sanitarias con mayores impactos producidos a lo largo de la historia, una pandemia ocasionada por el virus COVID-19 que ha sido capaz de provocar una irrupción en la economía global; la cual, no solo ha dejado pérdidas millonarias, sino, que ha desnudado las grandes diferencias y deficiencias de los sistemas en todos los sectores, entre ellos educación y salud. A ello, se le suma las restricciones producto de las medidas sanitarias adoptadas por las diversas autoridades gubernamentales de la región; por esta razón, se han "reestructurado" hábitos, se han cambiado rutinas para poder adaptarse a este nuevo y desafiante escenario. 
Latinoamérica, considerada como una de las regiones con mayor desigualdad social (CEPAL, 2016), a pesar de contar con países catalogadas con economías emergentes, ha sido el escenario idóneo para que las diferencias socioeconómicas se acentuaran durante este periodo de pandemia (Tejedor et al., 2020; Arriagada, 2020). El limitado y excluyente acceso a un adecuado sistema sanitario y educativo, el cual dependía de los recursos económicos del ciudadano, ha evidenciado no solo las deficiencias, sino las inadecuadas políticas implementadas dentro del marco de los Objetivos de Desarrollo Sostenible (ODS) (Jaramillo y Ñopo, 2020; Llerena y Sánchez, 2020).

Los confinamientos decretados por los diversos gobiernos, acompañados de sus normas de conducta y restricciones a todos los niveles, han producido, en la población, impactos respecto a su salud mental: depresión, ansiedad y los cambios en los hábitos de sueño, alimentación entre otros (Vásquez et al., 2020; Huarcaya-Victoria, 2020; Saravia-Bartra et al., 2020; Nascimento et al., 2020). En el sector educativo, esto se ha visto reflejado en las múltiples investigaciones (Arias et al., 2020; Anaya, 2020; Fernández, 2020), donde se evidencia que el estrés ha impactado la psique del estudiante, haciendo que este pierda la motivación en su rendimiento académico y cognitivo; y donde ha primado la ansiedad (González, 2020) sobre todo en aquellos que cuentan con menos recursos. En ellos, sus prioridades han cambiado, sus preocupaciones se han centrado, básicamente, en la situación laboral, la disponibilidad de materiales para poder atender sus necesidades, la conexión a internet, entre otros (Reyes y Trujillo, 2020; Ferri et al., 2020).

En este mismo contexto, debemos resaltar la importancia y el protagonismo que han tenido las TIC's, las cuales se han convertido en los elementos fundamentales para poder desarrollar la interacción y continuar con la formación académica. No obstante, no todas las instituciones contaban con la tecnología, estrategias, diseños curriculares y plataformas que permitían al estudiante afrontar esta situación de educación remota por emergencia. Las dificultades no solo estuvieron en el campo tecnológico, sino, también en el factor humano: los docentes, quienes se vieron obligados a capacitarse y aprender, en el camino, de las nuevas formas de comunicación y clases (Ferri et al., 2020; Toquero, 2020; Del Arco et al., 2021).

En este punto, es importante destacar el estudio realizado por Fardoun et al. (2020), quienes, a través de un estudio exploratorio y una participación de 102 docentes de Iberoamérica, indagaron las deficiencias y principales problemas que encontraron en países latinoamericanos para enfrentar la educación durante la pandemia. Así, es significativo que en su estudio nos detalle que países como Perú, México y Ecuador señalen que su principal problema se encontraba en la carencia de recursos tecnológicos, falta de plataformas tecnológicas (principalmente en México y Colombia) y desconocimiento de modelos pedagógicos (sobre todo en Perú para desarrollar una educación-enseñanza remota).

La educación remota por emergencia fue el camino por el cual se pudo mantener las sesiones de aprendizaje en todas las instituciones educativas. Con el establecimiento del aislamiento social obligatorio, debido a la pandemia, la educación universitaria, y en general, se vio forzada a migrar de la clase presencial a una educación en casa, de forma invasiva. Esta migración nos enfrentó con un nuevo proceso: el de una nueva enseñanza remota, en la cual los estudiantes se vieron obligados a adaptarse a un contexto ajeno a su formación previa. Se crearon nuevas exigencias en los actores del ámbito educativo. En el caso del docente, existió la necesidad 
de adaptarse a un nuevo proceso en el que debía impartir una sesión de clase a través de canales que quizá no había trabajado o desconocía como son Zoom, Teams de Microsoft, Meet de Google, Blackboard, entre otros. Se tuvo que capacitar en el empleo de estas herramientas, así como rediseñar parte de su programa académico. Mientras que, en el caso de los estudiantes, existió la necesidad de enfrentar un proceso de autonomía en su aprendizaje (Araujo et al., 2020). El alumno se enfrentaba a un escenario en el que no compartía un espacio ni con sus compañeros, ni con el docente.

Fue un concepto novedoso para quienes no habían implementado o asistido a una educación virtual, híbrida o blenderizada, las cual ya contaban con experiencia y estructura orgánica que permitía el desarrollo de una clase on line (Hodges et al., 2020). Es por esta razón que no podemos confundir el proceso de enseñanza llevado a cabo durante 2020-2021 con el de una educación virtual. En este último caso, la comunidad académica, en general, conoce la dinámica que se establece desde el inicio. Los actores involucrados en este tipo de formación académica saben a qué se enfrentan, atienden las exigencias y están preparados para afrontar una experiencia educativa de este nivel. En el caso de docentes y estudiantes, se exige la preparación y conocimiento previo en el empleo de equipos tecnológicos y plataformas educativas que dinamizan el proceso de enseñanza-aprendizaje.

En esta línea de investigación, el presente estudio surge de la necesidad de conocer cuáles fueron los efectos de este tipo de enseñanza (remota por emergencia) en la autonomía de los estudiantes universitarios. Los objetivos que se persiguen son analizar las experiencias desde diversos actores involucrados respecto a la enseñanza remota en universidades de Latinoamérica y analizar los resultados de investigaciones sobre el impacto de estas experiencias en la autonomía para el aprendizaje.

\section{Metodología}

Esta investigación está enmarcada en un enfoque cualitativo, basada en el análisis de las fuentes secundarias bajo un contexto específico (Slaw, 1999). En este caso se han seleccionado investigaciones publicadas en revistas indexadas en bases de datos abiertas como Scielo, Redalyc, Dialnet y Google Académico. Se tuvo como criterios de inclusión: geográficamente a universidades de Latinoamérica y la delimitación temporal dentro del periodo pandémico (20202021); además, se seleccionaron artículos escritos en español. Con la información procesada, se interpretaron y compararon los resultados en función a los objetivos específicos planteados: analizar las experiencias de la enseñanza remota en universidades de Latinoamérica, y el análisis de los impactos identificados de la enseñanza remota en la autonomía del aprendizaje. De esta manera, concordamos con Ruiz (2012), quien sostiene que el análisis conlleva a la interpretación de los documentos a través de la observación orientada a los objetivos planteados.

\section{Resultados}

\section{Experiencias en Latinoamérica}


A pesar de los intentos de parte de las universidades por adaptar el proceso de enseñanza presencial a la remota, los resultados no fueron las esperados. Ello dependió del uso de las TICs, los cuales ocuparon un rol relevante durante este proceso, puesto que debía encontrarse un equilibrio entre las competencias tecnológicas y académicas. Esto convirtió, a estas herramientas tecnológicas, en un medio del aprendizaje para alcanzar los objetivos en el que el docente debía cumplir un rol supervisor y de acompañamiento, mientras que el estudiante debía asumir un rol activo evidenciando su responsabilidad y autonomía (Ureta y Rossetti, 2020).

Además, la infraestructura, el liderazgo y la gestión desarrollada por las autoridades no se dieron en las mejores condiciones posibles para poder ejecutar una transición hacia la virtualidad más inclusiva y equitativa (Araujo et al., 2020); razón por la cual se llegó a una disconformidad por parte de los estudiantes. Un ejemplo de ello lo podemos encontrar en el artículo de Romero (2021), quien encontró cierta resistencia por parte de los alumnos pertenecientes al programa de educación andragógica, quienes consideraron que la presencialidad le ofrecía una mejor experiencia debido a la asistencia del docente a su lado y el conocimiento y familiaridad con las TIC's.

Por otro lado, ente los resultados que se tuvieron se señalan que el aula invertida, como un proceso de enseñanza en el que las metodologías activas promueven el aprendizaje autónomo, permitieron aprovechar al máximo el uso de los TIC's para lograr las competencias planteadas (Ventosilla et al., 2021). Dicha modalidad le permite al estudiante enfrentar los retos alrededor de su proceso de aprendizaje con el uso adecuado de los medios digitales, permitiéndoles afianzar su autonomía; sin embargo, las investigaciones también sostienen que dicho proceso debe permitir que el docente replantee su flexibilidad para promover la autorregulación (Peinado, 2020).

La adaptación de espacios adecuados dentro de los hogares, el uso obligatorio de la tecnología en la rutina académica y, sobre todo, el desarrollo de habilidades para afrontar estas nuevas condiciones cargadas de emociones como: paciencia, tolerancia y la flexibilidad, convirtieron a la enseñanza virtual en condiciones de confinamiento en un "cocktail" que presagiaba impactos en la comunidad educativa (Barros y Da Costa, 2021). El uso de las TICs no solo ha permitido que el proceso de enseñanza continúe a pesar de sus limitaciones en los países de la región; sino, también abrió nuevos espacios de interacción, en el que el tiempo y el espacio pueden permitirles a los actores del proceso a encontrar mecanismos para alcanzar los objetivos debido a su nueva condición de flexibilidad que ofrece (Villarroel y Ángeles, 2020).

Las experiencias de este nuevo escenario desencadenaron emociones para el docente y los estudiantes. Un escenario en el que los silencios incómodos, la limitada participación y la pérdida del interés fue convirtiéndose en una constante (Barros y Da Costa, 2021). Ello obligó a que se rediseñen no solo los materiales, sino, también las estrategias que motiven al estudiante, puesto que esto tendría un efecto positivo en su rol activo y autónomo en el proceso educativo (Mendoza y Vázquez, 2020). Sin embargo, no solo el dominio de nuevas herramientas tecnológicas y su adecuado uso son suficientes; es necesario repensar qué estrategias evaluativas pueden darse, lo cual conlleva a que se evalúen de forma integral los planes de estudios y se reestructure la organización y el proceso en su conjunto (Fardoun et al., 2020). Según De Sousa et al. (2020), los docentes y los estudiantes deben entender que, dentro de este marco de enseñanza, es necesario 
estructurar y planificar en función a las nuevas formas y condiciones, solo ello podrá asegurar la transferencia de una enseñanza presencial a una acorde con el contexto pandémico.

Otro lado del proceso de la educación en tiempos pandémicos está referido al lado humano del mismo; es decir, a las necesidades básicas por cubrir de los estudiantes, la cual los obliga a trabajar de forma virtual, y la sobrecarga del docente, trazado por la preparación de sus clases y por capacitarse para llevarlas a cabo dentro de dicho contexto. A todo esto, se le suma las limitaciones establecidas por los gobiernos de la región, las cuales obligan a las instituciones a repensar sus estrategias frente a esta situación. Es ante esta realidad que es necesario formular propuestas inclusivas y democráticas teniendo como base el fin supremo de las mismas, el cual es su compromiso con la sociedad y la investigación (Santos, 2020), y la adaptación de cambios en función al contexto (Saraiva et al., 2020).

Uno de los grandes retos a los que estuvieron sometidos los docentes fue la evaluación y la toma de decisiones respecto al traslado de las prácticas presenciales al mundo virtual, en el que era necesario tomar en cuenta las formas y códigos de interacción en red (Schwartzman et al., 2021). Dicha modalidad ha provocado que los docentes consideren al incremento de las actividades como la mejor respuesta a los nuevos escenarios, ocasionando una sobrecarga laboral y una mayor exposición frente a la computadora. Sin embargo, ello se ha visto reflejado inversamente proporcional a la interacción y participación del estudiante, lo que a su vez produce un desgaste para ambas partes. Se desaprovecha el momento que deberían verse como una oportunidad para abrir espacios de diálogo que tengan como resultado una propuesta real al sector educativo que ha visto desnudadas sus falencias (Silas y Vázquez, 2020).

Los estudios latinoamericanos enfocados en comprender dicho proceso de enseñanza remota han tenido algunas características que deben considerarse. A nivel metodológico, se tuvieron artículos con enfoque cuantitativo de diseño experimental (Romero, 2021; Santamaría et al., 2020), no experimental (Appenzeller et al., 2020; Niño et al., 2021; Portillo et al., 2020; Rueda, 2020); trabajos con enfoque cualitativo (De Sousa et al., 2020; De França y De Freitas, 2020; Silva et al., 2021; Aboes, 2020; Pereira et al., 2020) y, finalmente, también se encontraron trabajos con diseño mixto (Andrade et al., 2021; Ries et al., 2020). Estas fuentes tuvieron en común que dichos análisis se basaron desde la perspectiva de los actores claves: docentes y estudiantes; por ello, los objetivos predominantes estaban orientados a medir la percepción y analizar las propuestas planteadas. En cuanto a las conclusiones a las que llegaron, pueden orientarse básicamente a tres; la primera vinculada con la percepción positiva por parte de los actores debido al rol de las instituciones y a las ventajas que puede ofrecer esta modalidad. La segunda orientada a la importancia y relevancia que tiene contar con un adecuado uso de capacitación e inducción por parte de los actores respecto al uso de TIC's, el cual debe ir acompañado con el acceso al servicio de internet. Y la tercera, en que la negatividad estuvo presente en la percepción de los actores respecto a la experiencia de aprendizaje remota. 
Figura 1

Impactos de la Enseñanza Remota según análisis de investigaciones

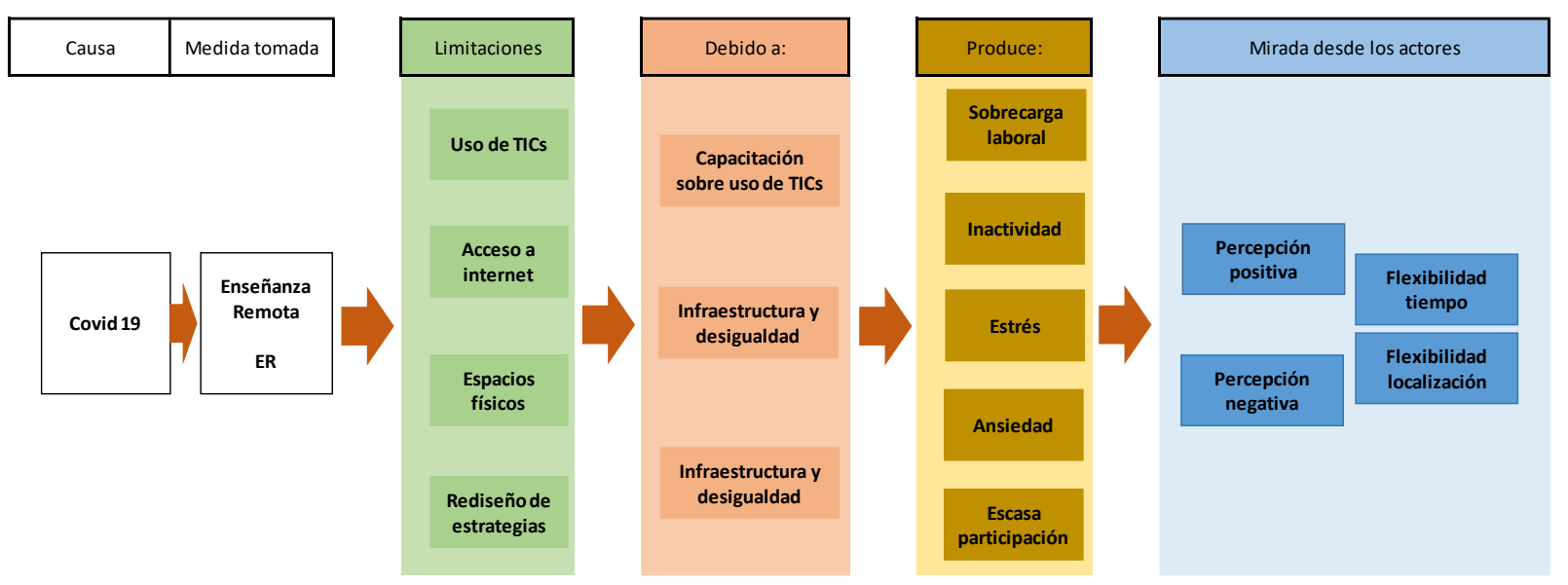

Nota: Esquema elaborado como resultado del análisis de las investigaciones

\section{Impactos en la autonomía del aprendizaje}

Respecto a los impactos de la adaptación de sesiones remotas en la autonomía para el aprendizaje de los estudiantes, los resultados están sujetos a lo que las instituciones o docentes, como iniciativa individual, hayan realizado respecto al diseño instruccional. Este último debe ser entendido como la planeación del acto educativo: el diseño de actividades, la aplicación de estrategias, técnicas, la elaboración de recursos, la forma de evaluación, la retroalimentación; es decir, la planificación del proceso de aprendizaje en un entorno virtual. En esa línea, Martín y Salcedo (2018), sostienen que los procesos formativos mediados por tecnología, que buscan el desarrollo de competencias superiores, deberán prever el diseño para la instrucción, el acompañamiento del docente, así como un proceso evaluativo que promuevan en desarrollo de habilidades relacionadas a la autogestión de los aprendizajes, enfatizando el rol relevante de la planificación, lejos de sostener que la educación a distancia solo por su naturaleza permite una gestión flexible del tiempo y que otorga autonomía a los estudiantes, tal como sostienen Perez et al. (2020). Sobre esto, Venosa (2020) sostiene que formar estudiantes en situación de emergencia implica entonces una gran responsabilidad por parte del docente, para que a través del diseño de clase y su guía, los estudiantes adquieran estrategias y desarrollen habilidades para la gestión de sus propios aprendizajes.

En ese marco, los estudios que se muestran a continuación, evidencian diferentes tipos de acciones por parte de los docentes y sus implicancias en el aprendizaje autónomo. Se buscaron y emplearon estrategias que permitieran el desarrollo de la autonomía en un contexto no planificado (Araujo et al., 2020). Así, Peinado (2020) realizó un estudio con profesores de educación a distancia de un instituto, encontró que cuando el docente, previo análisis del propósito educativo y posterior generación de diversos recursos digitales, logra que los estudiantes utilicen estos recursos para aprender por sí mismos, promoviendo el pensamiento crítico y reflexivo, conocimientos significativos, habilidades meta cognitivas; así como la autorregulación de los 
aprendizajes. Así mismo, Nieves et al. (2021), usaron la tele cirugía y los videos para desarrollar el aprendizaje autónomo y competencias en el área médica, de tal manera que los estudiantes no vean sujeta su formación a la presencia física en el laboratorio o en el hospital en contexto del COVID-19. Hallazgos como los de Mendoza y Vásquez (2020) muestran, además, que la retroalimentación instantánea, un formato atractivo y la visualización dinámica de las actividades, promovieron que el estudiante se motive a seguir aprendiendo superándose a sí mismo y en relación al grupo.

En esa misma línea, Ventosilla et al. (2021), en un estudio aplicado en Perú, lograron desarrollar el aprendizaje autónomo, promover el aprendizaje colaborativo y reforzar aspectos afectivos y actitudinales haciendo uso de metodologías activas, mediante la aplicación de la metodología de aula invertida. Aclaran que para desarrollar una autonomía del aprendizaje es necesario que el docente se adecúe a los cambios que implica el empleo de la tecnología y se adapte a facilidades que esta le ofrece para enriquecer el desarrollo de enseñanza aprendizaje y, sobre todo, considerar las particularidades y ritmos de aprendizaje de los estudiantes. Este aspecto comunicacional y de soporte emocional, con docentes empáticos ante diversas problemáticas, son para Galindo, et.al., (2020) fundamentales para fortalecer la autonomía de los estudiantes. En ese sentido Ureta y Rossetti (2020), refieren que la incorporación de prácticas pedagógicas contextualizadas deben tener como propósito principal la construcción de aprendizajes significativos, indicando que para ello se debe tener claridad sobre el rol que desempeñan los docentes y estudiantes en entornos virtuales: el docente como guía, orientador, supervisor, motivador constante y evaluador, y el estudiante asumiendo un rol autónomo, que debe buscar desarrollar habilidades para la autogestión de sus aprendizajes.

Los resultados mostrados, hacen ver que el uso de estrategias metodológicas, recursos virtuales y evaluación pertinente, en la que tiene mayor importancia la retroalimentación, son, desde la práctica, herramientas útiles para "aprender a aprender". Este proceso debe entenderse como una habilidad compleja superior a la meta cognición, la cual abarca procesos cognitivos, motivacionales, emocionales y aspectos del medio, que permiten que las personas en general podamos continuar aprendiendo de forma cada vez más eficaz e independiente, de acuerdo a nuestros propios propósitos y necesidades (Patarroyo y Navarro, 2017). Evidentemente, estos conceptos también aplican a los docentes y su renovación continua de la enseñanza-aprendizaje.

\section{Figura 2}

Impactos de la enseñanza remota en la autonomía del aprendizaje

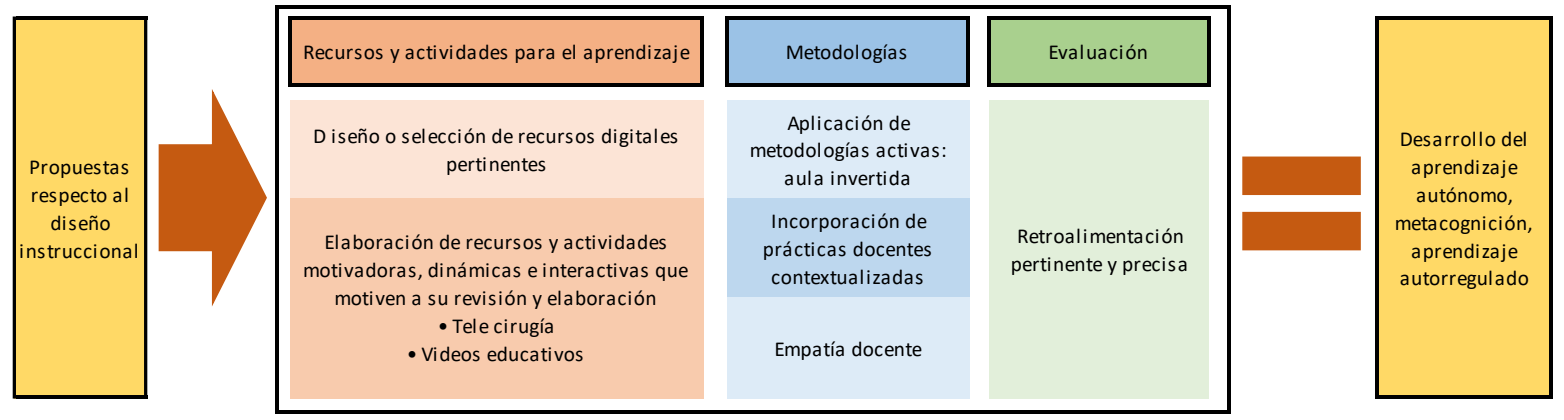




\section{Conclusiones}

La educación y enseñanza remota debido a la emergencia sanitaria, han repercutido enormemente en la educación superior en América Latina. Las evidencias, de las fuentes consultadas, nos advierten que las instituciones educativas no estuvieron preparadas para enfrentar un escenario de estas características y que enfrentaron dificultades a nivel estructural y pedagógico. En el primer campo, se sostienen las dificultades en el empleo de recursos para un buen desarrollo educativo que contrasta con la experiencia presencial. En cuanto al segundo, las grandes deficiencias estuvieron dadas por el diseño pedagógico para establecer nuevas estrategias de enseñanza-aprendizaje que permita al estudiante no solo comprender, sino, también para desarrollar una autonomía propia del aprendizaje a nivel superior.

El presente estado del arte se enfocó en dos objetivos esenciales derivados de esta enseñanza remota por emergencia. La primera estuvo guiada por el análisis de las experiencias que se dieron en la enseñanza superior desde espacios latinoamericanos. En ella podemos concluir que las dinámicas desarrolladas para una sesión de clase no sufrieron cambios significativos, se optó por trasladar una educación presencial en el aula, a una virtual con el empleo de una cámara. El paso de una experiencia a otra no significó una nueva percepción en cuanto a la metodología de enseñanza-aprendizaje, no hubo un cambio significativo y se mantuvo el status quo de una modalidad presencial; se migró sin una secuencia que permita entender y mejorar este sistema. No obstante, se encontró también que este cambio, en el empleo de los canales de comunicación (clase remota), desmotivaron a los estudiantes y provocaron una sobrecarga en cada uno de los actores involucrados. En el caso de docentes, tuvo la necesidad de capacitarse y al mismo tiempo se llenó y saturó de evaluaciones y/o actividades, esto último también en los estudiantes.

Es importante, también, resaltar la utilidad de las TIC's, que ayudaron en el desarrollo de clase, las nuevas tecnologías aportaron significativamente y serán de gran utilidad en el futuro para complementar la información desarrollada en una sesión de clase cuando se regrese a la presencialidad. Se debe tomar en cuenta las capacitaciones desarrolladas en este campo para repotenciar y mejorar las dinámicas y estrategias de enseñanza, lo cual permitirá alcanzar logros significativos en los estudiantes.

El segundo objetivo estuvo orientado en conocer y analizar la autonomía del aprendizaje de los estudiantes a partir de esta enseñanza remota por emergencia. Se destacan los grandes esfuerzos y responsabilidades del docente para lograr que los estudiantes "aprendan a aprender". La empatía, el reconocimiento del ritmo de aprendizaje de los alumnos, sumado al empleo de estrategias, recursos tecnológicos y una buena retroalimentación, posibilitan el desarrollo de la autonomía del aprendizaje en el estudiante. Se deben considerar diversos factores que como docentes no se pueden controlar al desarrollar una clase virtual, es por ello que aspectos afectivos y emocionales, los buenos vínculos y la adecuada comunicación que genere el docente, son valorados como importantes y positivos por los estudiantes. 


\section{Referencias bibliográficas}

Aboes, L. (2020). Aulas Remotas em tempos de COVID-19: a Percepção de discentes de um programa de mestrado profissional em educação. Revista @ mbienteeducação, 13(2), 4760. https://doi.org/10.26843/ae19828632v13n22020p47a60

Andrade, E., Furlan, G., Dutra, J., y Renda, L. (2021). Experiencias com o ensino remoto e os efeitos no interesse e na satisfacao dos estudantes de ciencias contabies durante a pandemia da SARS-CoV-2. Revista Gestao Organizacional, 356-377. https://doi.org/0.22277/rgo.v14i1

Anaya, J. P. (2020). Ansiedad y agresividad en tiempos de pandemia-Covid 2019 en Lima Metropolitana (Tesis de licenciatura, Universidad César Vallejo, Facultad de Ciencias de la Salud. Lima, Perú). https://hdl.handle.net/20.500.12692/56820

Appenzeller, S., Menezes, F. H., Santos, G. G. dos, Padilha, R. F., Graça, H. S., y Bragança, J. F. (2020). Novos Tempos, Novos Desafios: Estratégias para Equidade de Acesso ao Ensino Remoto Emergencial. Revista Brasileira de Educação Médica, 44(suppl 1), 1-6. https://doi.org/10.1590/1981-5271v44.supl.1-20200420

Araujo, L., Ochoa, J., y Vélez, C. (2020). El claroscuro de la universidad ecuatoriana: los desafíos en contextos de la pandemia de COVID-19. Revista Digital de Investigación En Docencia Universitaria, 14(2), e1241. https://doi.org/10.19083/10.19083/ridu.2020.1241

Arias, Y., Herrero, Y., Cabrera, Y., Chibá, D., y García, Y. (2020). Manifestaciones psicológicas frente a la situación epidemiológica causada por la COVID-19. Revista Habanera de Ciencias Médicas, 19 (Supl. 1) https://bit.ly/3B8cj2Q

Arriagada, P. (2020). Pandemia COVID-19: educación a distancia. O las distancias en la educación. Revista Internacional de Educación Para La Justicia Social, 9(3). https://revistas.uam.es/riejs/article/download/12396/12222/32897

Barros, P., y Da Costa, J. (2021). Pedagogía en tiempos de pandemia: afectos y memorias de la enseñanza-aprendizaje. Digital Publisher, 6(2-1), 229-241. https://doi.org/10.33386/593dp.2021.2-1.505

CEPAL. (2016). La matriz de la desigualdad social en América Latina. In CEPAL (Vol. 38, Issue 3). https://bit.ly/3BbiumJ

De França, C., y De Freitas, L. (2020). Remoto, mas não distante: a reinvenção do ensino na times of COVID-19. Dialogia, 382-395. https://doi.org/10.5585/dialogia.n36.18828

Del Arco, I., Silva, P., \& Flores, O. (2021). University teaching in times of confinement: The light and shadows of compulsory online learning. Sustainability (Switzerland), 13(1), 1-16. https://doi.org/10.3390/su13010375

De Sousa, N., Batista, J., Cabral, M., Gomes, T., Amado, C., Dos Santos, R., y De Castro, E. (2020). Ensino remoto na enfermagem em meio a pandemia da COVID-19. Revista Científica ENFERMAGEM, 10(32), 358-366. https://doi.org/10.24276/rrecien2020.10.32.358-356

Fardoun, H., González, C., Collazos, C., y Yousef, M. (2020). Estudio exploratorio en Iberoamérica sobre procesos de enseñanza-aprendizaje y propuesta de evaluación en tiempos de pandemia. Education in the Knowledge Society, 21, 171-179. https://doi.org/10.14201/eks.23437

Fernández A. (2020). 2020: Estudiantes, emociones, salud mental y pandemia. Revista Andina De Educación, 4(1), 23-29. https://doi.org/10.32719/26312816.2021.4.1.3

Ferri, F., Grifoni, P., \& Guzzo, T. (2020). Online Learning and Emergency Remote Teaching: 
Opportunities and Challenges in Emergency Situations. Societies, 10(4), 86. https://doi.org/10.3390/soc10040086

Galindo, D. García, L., García, R., Gonzales, P., Hernández, P. López, M., Luna, V. y Moreno, C. (2020). Recomendaciones didácticas para adaptarse a la enseñanza remota de emergencia. Revista Digital Universitaria. http://doi.org/10.22201/cuaieed.16076079e.2020.21.5.15

González, L. (2020). Estrés académico en estudiantes universitarios asociados a la pandemia por COVID-19. Espacio I+D, Innovación más Desarrollo, 9 (25). https://doi.org/10.31644/IMASD.25.2020.a10

Hodges, C., Lockee, B., Moore, S., \& Trust, T. (2020). View the full-text article online: March. https://bit.ly/3mhu4Zt

Huarcaya-Victoria, J. (2020). Consideraciones sobre la salud mental en la pandemia de COVID19. Revista Peruana de Medicina Experimental y Salud Pública, 37(2), 327-334. https://doi.org/10.17843/rpmesp.2020.372.5419

Jaramillo, M., y Ñopo, H. (2020). El impacto del COVID-19 sobre la economía peruana. Economía UNAM, 17(51), 136-146. https://bit.ly/3bgkcbT

Llerena, R., y Sánchez, C. (2020). Emergencia, gestión, vulnerabilidad y respuestas frente al impacto de la pandemia COVID-19 en el Perú, 16. https://preprints.scielo.org/index.php/scielo/preprint/view/94/129

Martín, A. y Salcedo, E. (2018). La pertinencia de enseñar a aprender estratégicamente en el nivel de posgrado. Revista de Humanidades. 33 (2018), 87-114. https://doi.org/10.5944/rdh.33.2018.18293

Mendoza, A., y Vázquez, V. (2020). REA para el aprendizaje a distancia que promueve la motivación y la autonomía. Revista Lengua y Cultura, 2(3), 1-7. https://repository.uaeh.edu.mx/revistas/index.php/lc/article/view/6183.

Nascimento, H., Santos, L., y Olveira, A. (2020). Efeitos da pandemia do novo Coronavírus na saúde mental de indivíduos e coletividades Após análise dos exames, um novo do uso de sequenciamento genético pacientes, que foram usadas para um antes, com registro da morte no dia COVID-19 disponibilizado no. Jorunal of Nurisgn and Heath, 10. https://doi.org/e20104007

Nieves, B., Rodríguez, J. y Somoano, A. (2021). Adquirir habilidades quirúrgicas en tiempos de pandemia: la telecirugía y el aprendizaje autónomo con videos. Fundación educación médica. 24(2), 107-108. https://bit.ly/3jxpF2v

Niño, S., Castellanos, J., y Patrón, F. (2021). Contraste de experiencias de estudiantes universitarios en dos escenarios educativos: enseñanza en línea vs. enseñanza remota de emergencia Contrasting the experiences of university students in two educational scenarios: online teaching vs. emergency. Revista de Educación a Distancia, 21(65), 1-25. https://doi.org/10.6018/red.440731

Pararroyo, C., y Navarro, M. 2017). Aprender a Aprender: La apuesta pedagógica de la Universidad del Rosario Mesa de reflexión y de fomento a la docencia de excelencia en la Universidad del Rosario. Reflexiones pedagógicas Urosario. (9). https://editorial.urosario.edu.co/pageflip/acceso-abierto/reflexiones-pedagogicas-9.pdf

Peinado, J. (2020). Experiencias del profesorado acerca del aprendizaje autónomo en estudiantes de modalidad a distancia y el uso de recursos digitales. RIDE Revista Iberoamericana Para La Investigación y El Desarrollo Educativo, $10(20)$. https://doi.org/10.23913/ride.v10i20.645 
Pereira, D., Rodrigues, N., \& Vargas, S. (2020). Os refl exos do ensino remoto na docência em tempos de pandemia da COVID-19. EDaPECI, 72-86. https://doi.org/10.29276/redapeci.2020.20.314543.72-86

Perez, E. Vásquez, A. y Cambero, S. (2020). Educación a distancia en tiempos de COVID-19: Análisis desde la perspectiva de los estudiantes universitarios. Revista Iberoamericana de Educación a Distancia. 24(1), 331- 342. http://doi.org/10.5944/ried.24.1.27855

Portillo, S., Castellanos, L., Reynoso, O., y Gavotto, O. (2020). Enseñanza remota de emergencia ante la pandemia COVID-19 en Educación Media Superior y Educación Superior. Propósitos $\quad y \quad$ Representaciones, $\quad 8(3), \quad 1-17$. https://doi.org/10.20511/pyr2020.v8nSPE3.589

Reyes N. \& Trujillo P. (2020). Ansiedad, estrés e ira: el impacto del COVID-19 en la salud mental de estudiantes universitarios. Investigación \& desarrollo, 1(13) https://bit.ly/2ZqD6uD

Ries, E., Rocha, V., \& Lopes, C. (2020). Avaliação do ensino remoto de Epidemiologia em uma universidade pública do Sul do Brasil durante pandemia de COVID-19. https://doi.org/10.1590/SciELOPreprints.1152

Romero, V. (2021). Disrupción de la educación remota en el programa universitario para adultos en tiempos de COVID-19. Apuntes Universitarios, 11(1), 401-414. https://doi.org/10.17162/au.v11i1.593

Rueda, K. (2020). Estrategia educativa remota en tiempos de pandemia. Magister, 32(1), 93-96. https://doi.org/10.17811/msg.32.1.2020.93-96

Ruiz, J. (2012). Metodología de la Investigación. Universidad de Deusto. Bilbao

Santamaria, T., Lagos, G., y González, V. (2020). Importancia de la tecnología de transmisión para teleenseñanza/ enseñanza remota: evaluación de la aplicación a un curso de tecnología de la información. RISTI: Iberian Journal of Information Systems and Technologies, 31, 404422. https://bit.ly/3vQ8T3z

Santos, B. (2020). Educação No Ensino Superior Em tempos de pandemia. Olhar de Professor, 23. https://doi.org/10.5212/OlharProfr.v.23.2020.16175.209209226867.0616

Saraiva, K., Traversini, C., y Lockmann, K. (2020). La educación en tiempos de COVID-19: enseñanza remota y agotamiento docente. Práxis Educativa, 15(1809-4031), 1-24. https://doi.org/10.5212/PraxEduc.v.15.16289.094

Saravia-Bartra M., Cazorla-Saravia P., \& Cedillo-Ramirez L. (Octubre,2020). Anxiety level of first-year medical students from a private university in Peru in times of COVID-19. Revista de la Facultad de Medicina Humana, 20(4), 568-573. https://doi.org/10.25176/RFMH.v20i4.3198

Schwartzman, G., Roni, C., Berk, M., Delorenzi, E., Sánchez, M., y Eder, M. (2021). Evaluación Remota de Aprendizajes en la Universidad: decisiones docentes para encarar un nuevo desafío. RIED. Revista Iberoamericana de Educación a Distancia, 24(2), 67. https://doi.org/10.5944/ried.24.2.29078

Silas, J., y Vázquez, S. (2020). El docente universitario frente a las tensiones que le plantea la pandemia. Resultados de un estudio mexicano/latinoamericano. Revista Latinoamericana de Estudios Educativos, $\quad$ L(Especial), $\quad$ 89-119. https://doi.org/10.48102/rlee.2020.50.ESPECIAL.97

Shaw, E. (1999). A guide to the Qualitative Research Process: Evidence from a Small Firm Study. Qualitative Market Research: An International Journal, 2 (2): 59-70. https://bit.ly/3bbFQy4

Silva, C., Tamami, A., Toriyama, M., Claro, H., Amaral, C., Castro, T., Ivo, P., \& Alves, C. (2021). 
Pandemia da COVID-19, ensino emergencial a distância e Nursing Now: desafios à formação em enfermagem. https://doi.org/10.1590/1983-1447.2021.20200248

Tejedor, S., Cervi, L., Tusa, F., y Parola, A. (2020). Educación en tiempos de pandemia: reflexiones de alumnos y profesores sobre la enseñanza virtual universitaria en España, Italia y Ecuador. Revista Latina de Comunicación Social, 78, 1-21. https://doi.org/10.4185/rlcs-2020-1466

Toquero, C. (2020). Emergency remote education experiment amid COVID-19 pandemic. IJERI: International Journal of Educational Research and Innovation, 15, 162-176. https://doi.org/10.46661/ijeri.5113

Ureta, L., y Rossetti, G. (2020). Las TAC en la construcción de conocimiento disciplinar: una experiencia de aprendizaje con estudiantes universitarios. Revista Iberoamericana de Tecnología En Educación y Educación En Tecnología, 26, e11. https://doi.org/10.24215/18509959.26.e11

Vásquez, G., Urtecho-Osorto, O., Agüero-Flores, M., Díaz, M., Paguada, R., Varela, M., LandaBlanco, M., y Echenique, Y. (2020). Salud mental, confinamiento y preocupación por el coronavirus: un estudio cualitativo. Revista Interamericana de Psicología/Interamerican Journal of Psychology, 54(2), e1333. https://doi.org/10.30849/ripijp.v54i2.1333

Venosa, H. (2020). Desarrollo y promoción de la autonomía durante el trabajo remoto por la COVID-19 con adolescentes. https://bit.ly/3jy5zWc

Ventosilla, D., Santa María, H., De La Cruz, F., y Flores, A. (2021). Aula invertida como herramienta para el logro de aprendizaje autónomo en estudiantes universitarios. Propósitos $\quad y \quad$ Representaciones, $\quad 9(1), \quad$ 1043-undefined. https://doi.org/10.20511/pyr2021.v9n1.1043

Villarroel, P., y Ángeles, M. D. L. (2020). Implementación de curso on line Anatomía y la percepción de los estudiantes de Kinesiología. Inv Ed Med, 9, 75-84. https://www.medigraphic.com/pdfs/invedumed/iem-2020/iem2035i.pdf 